\title{
A retrospective cohort study of patients treated with anti-tuberculous therapy for presumed ocular tuberculosis
}

\author{
Erika Marie Damato', Sarah Dawson ${ }^{1}$, Xiaoxuan Liu², Chandoshi Mukherjee ${ }^{1}$, John Horsburgh ${ }^{3}$, \\ Alastair K. Denniston ${ }^{3,4}$, Edward Moran ${ }^{5}$, Martin Dedicoat ${ }^{6}$ and Philip lan Murray ${ }^{1,4^{*}}$
}

\begin{abstract}
Background: Uveitis involving the posterior segment is a significant and potentially blinding condition. The diagnosis and treatment of patients with uveitis associated with tuberculosis remains controversial, and commonly, patients are systemically well. Use of the interferon-gamma release assays has added to the controversy, as the significance of a positive test may be uncertain. We aim to report the outcomes of anti-tuberculous treatment in a cohort of patients treated in Birmingham, for presumed "ocular tuberculosis", based on clinical findings, systemic assessment and specific testing for tuberculosis.
\end{abstract}

Results: We found that in our cohort of 41 patients treated between 2010 and 2014, the majority achieved diseasefree remission, even in cases where anti-tuberculous treatment was delayed.

Conclusions: Despite controversy, this study strongly supports the use of anti-tuberculous therapy in such patients and highlights the need for formal prospective trials and treatment protocols.

Keywords: Uveitis, Anti-tuberculous therapy, Tuberculosis, Interferon-gamma release assay

\section{Background}

Intraocular inflammation, generally termed "uveitis", may result from a hugely diverse range of causes, both infectious and non-infectious. Uveitis is classified by the predominant anatomical site of inflammation within the eye [1], the cause and whether or not it is related to an infectious agent or an underlying systemic condition [2]. Uveitis is an important cause of visual loss, often affecting the working age population $[3,4]$.

Uveitis related to tuberculosis is a well-recognized clinical entity, with an extensive literature dating back more than 100 years [5]. In some parts of the world, tuberculosis is one of the principle causes of uveitis [6-9].

Currently, a large proportion of patients in the developed world is described as having "non-infectious", "idiopathic" or

\footnotetext{
* Correspondence: p.i.murray@bham.ac.uk

'Birmingham and Midland Eye Centre, City Hospital, Sandwell and West Birmingham Hospitals NHS Trust, Birmingham B18 7QH, UK

${ }^{4}$ Institute of Inflammation and Ageing, College of Medical and Dental Sciences, University of Birmingham, Edgbaston, Birmingham B15 2TT, UK Full list of author information is available at the end of the article
}

"undifferentiated" uveitis $[10,11]$. Often no underlying systemic association or infection is identified despite extensive investigation. If such patients have significant or "sightthreatening" uveitis, they are generally treated with systemic corticosteroid, and sometimes systemic immunosuppression, including biologics $[12,13]$. Such therapy is aimed at preventing relapses, as each relapse carries a risk of irreversible visual loss, morbidity and ocular complications [14].

The association between uveitis and "latent" or occult tuberculosis is not new yet has become increasingly relevant. Many patients display clinical appearances identical to those seen in patients with active TB, without demonstrating systemic manifestations of infection. Ocular appearances associated with TB are heterogeneous, making diagnosis challenging, and include (among others) choroiditis, serpiginous-like choroiditis, granulomatous uveitis, retinal vasculitis and intermediate uveitis [15]. In addition, the condition known as "Eales" disease, a bilateral occlusive retinal vasculitis, with minimal inflammation and often complicated by vitreous haemorrhage, is observed more frequently in TB-endemic populations. 
A large proportion of the global population will have immunological evidence of latent TB, and consequently, a significant proportion of patients with ocular inflammation will have evidence of latent $\mathrm{TB}$, whether or not it is causal.

Studies investigating intraocular samples provide support for a central role of TB. Analysis of the aqueous humor, vitreous gel or epiretinal membranes using polymerase chain reaction (PCR) methods [16-20] demonstrates that a higher proportion of samples from uveitis patients are positive for the TB genome compared with samples taken from patients in a similar population undergoing surgery for other reasons, and a positive PCR result correlates with a clinical response to anti-tuberculous treatment (ATT) $[19,20]$.

Traditionally, the tuberculin skin test (Mantoux) has been used to determine whether a patient is infected with TB. The test may be affected by multiple factors, making it hard to determine its significance. More recently, interferon gamma release assays (IGRAs) have added significant controversy to the diagnosis and management of "ocular TB" [21-26]. Such assays, including the T-spot or QuantiFERON GOLD tests rely on the observation that T-cells will release interferon gamma when exposed to a specific TB antigen. The test is highly specific and not affected by previous BCG vaccinations. A positive IGRA test usually indicates that a patient has been exposed to TB; however, it cannot distinguish between latent infection and active disease.

Multiple recent reports support the use of antituberculous therapy (ATT) in patients with a consistent uveitic phenotype and positive TB investigations [27-30]. There is a lack of consensus regarding treatment indications, treatment regimens, investigation protocols and what constitutes a successful outcome. Even the diagnosis of "ocular TB" is not standardized [31].

Importantly, the way in which patients are managed is believed to differ significantly between units, with some uveitis specialists unable to persuade the respiratory physician of the relevance of tuberculosis, whilst other specialists are able to access anti-TB treatment easily and as a result have a very low treatment threshold [32]. Some uveitis specialists elect to refer patients for ATT only if the uveitis is severe, whilst continuing to treat "mild" recurrent anterior uveitis with topical steroid.

The principle indication for using ATT in uveitis is to treat the underlying systemic drive, which is believed to come from occult infection, antigenic mimicry or a hypersensitivity-type reaction to TB antigen. A secondary indication may be to ensure that systemic immunosuppression is safe in the face of a positive T-spot result. This is especially relevant for patients started with anti-TNF therapy [33].

The Birmingham and Midland Eye Centre is located in an inner-city part of Birmingham where the large surrounding population comprises of ethnically diverse communities with a majority of people originating from Southeast Asia, especially countries such as Pakistan, India and Bangladesh, where the prevalence of $\mathrm{TB}$ is high.

\section{Aims and objectives}

The aims of this study were first, to define and report the treatment outcomes of patients, who received antituberculous therapy for uveitis, and second, to characterize the patient cohort seen at the Birmingham and Midland Eye Centre who were diagnosed as having ocular TB.

\section{Methods}

This was a retrospective evaluation of patient notes. Ethical approval was not required as the project was deemed to be a service evaluation.

Using the regional TB disease register obtained from the regional infectious diseases department, patients who had been diagnosed as having uveitis related to TB and who were started on ATT between 2010 and 2014 were identified. Patients who completed a course of treatment and were followed up for at least 12 months were included in the study.

A retrospective analysis of patient notes was undertaken. Ethical approval was not required as this was deemed to be a service evaluation and made use of anonymized retrospective patient information. Data pertaining to patient characteristics, uveitis phenotype, vision, duration of uveitis prior to therapy, date of commencement of ATT, treatment details, duration of follow-up and disease activity were collected. Data were also collected regarding systemic investigations for TB.

At presentation to the uveitis service, patients underwent a full ophthalmic examination and were assessed with regard to the site and severity of their uveitis, using the SUN criteria [1]. A full systemic workup was undertaken, and patients underwent an extensive panel of investigations, with the aim of excluding other causes. All underwent chest radiograph or CT scan, with most also undergoing Mantoux testing or interferon gamma release assay, usually using T-spot. Investigations were not protocolized, and patients were investigated appropriately in an individualized fashion.

A consistent ocular phenotype, together with findings on chest imaging and/or positive T-spot or Mantoux testing, with the exclusion of other causes of uveitis, led to a diagnosis of presumed ocular TB.

Following this diagnosis, patients were referred urgently to the respiratory team where they were investigated further as necessary. Throughout the course of the study, the availability and use of interferon gamma release assays increased. This meant that some patients were not diagnosed with TB-related uveitis until a significant period of ocular inflammation had lapsed. 
In most patients, disease activity was observed as a number of relapses, rather than continual inflammation. Treatment with anti-inflammatory medication, mainly corticosteroids, was used. These were administered topically, peri-ocularly or systemically. Relapse on withdrawal of therapy warranted repeat treatment.

Ocular disease activity was quantified using the number of "flare-ups" of uveitis. A uveitis "flare" was defined as the need for augmentation of treatment, with high-dose oral steroid (usually at least $40 \mathrm{mg}$ daily tapered over several weeks), intravenous steroid, periocular steroid or hourly topical steroid. Where possible, "flare rate" prior to ATT was defined as the number of flare-ups per unit time of disease. Calculating a "flare rate" was not possible if a patient was treated with ATT immediately or had a very short duration of disease prior to ATT.

\section{Statistical methods}

Data were reviewed using descriptive statistics. The "time to flare" post-treatment was calculated using Kaplan-Meier survival curves. Remission was defined as the absence of flare-ups for at least 6 months whilst long-term remission was defined as at least 12 months of disease quiescence after completion of therapy. Flare rates were compared before and after therapy using the Wilcoxon signed rank test.

\section{Results}

\section{Demographics}

A total of 54 patients were identified as having uveitis related to ocular TB and for whom treatment was recommended. Of these, 41(76\%) patients completed therapy and complied with follow-up for at least 12 months. The remainder either did not complete therapy, did not comply with follow-up or both. The characteristics of the initial patient cohort are described in Table 1. The majority of patients were male, of Asian or Black ethnicity with a mean age of 44 years.

\section{Ocular disease}

Uveitis was predominantly bilateral with most having disease affecting the posterior segment. Isolated retinal vasculitis, including the "Eales' phenotype", was categorized as posterior uveitis. A wide range of uveitic phenotypes was observed, including granulomatous anterior uveitis, retinal vasculitis, nodular scleritis, choroiditis and intermediate uveitis.

The date of first presentation with uveitis preceded the diagnosis of TB-related eye disease in the majority, with four patients starting ATT at the same time as their uveitis was diagnosed. The duration of disease prior to ATT ranged from 8 to 4495 days (more than 12 years) with a median of 12 months. Prior to ATT, 31 patients were on maintenance therapy for uveitis as described in Table 1.
Table 1 Characteristics of the patient cohort

\begin{tabular}{|c|c|c|}
\hline \multicolumn{3}{|l|}{ Gender (number of patients (\%)) } \\
\hline Male & 33 & $61 \%$ \\
\hline Female & 21 & $39 \%$ \\
\hline Age, years, mean (range) & $44(17-69)$ & \\
\hline \multicolumn{3}{|l|}{ Ethnicity (number of patients (\%)) } \\
\hline Asian & 31 & $57 \%$ \\
\hline Black & 11 & $20.37 \%$ \\
\hline Not specified & 12 & $22.22 \%$ \\
\hline Diabetic (number of patients (\%)) & 8 & $14.81 \%$ \\
\hline \multicolumn{3}{|l|}{ Site of uveitis (number of patients (\%)) } \\
\hline Anterior & $6(11.11 \%)$ & \\
\hline Intermediate & $15(27.78 \%)$ & \\
\hline Posterior & $12(22.22 \%)$ & \\
\hline Panuveitis & 19 (35.19\%) & \\
\hline Other & $2(3.70 \%)$ & \\
\hline Bilateral disease (number of patients (\%)) & $41.58(77 \%)$ & \\
\hline \multicolumn{3}{|c|}{$\begin{array}{l}\text { Uveitis features present (NB some patients may have had more than } \\
\text { one feature) }\end{array}$} \\
\hline Nodular scleritis & 1 & \\
\hline Choroiditis & 7 & \\
\hline Granulomatous anterior uveitis/ mutton fat KP & 7 & \\
\hline Retinal vasculitis & 5 & \\
\hline Panuveitis & 13 & \\
\hline Intermediate uveitis & 18 & \\
\hline Not recorded & 3 & \\
\hline Maintenance treatment prior to ATT & Number of pa & atients (\%) \\
\hline Unknown & $8(14.80)$ & \\
\hline Nil & $13(24.10)$ & \\
\hline Topical steroid alone & $20(37)$ & \\
\hline Oral steroid alone & $3(5.60)$ & \\
\hline Oral steroid and immunosuppression & $2(3.70)$ & \\
\hline Topical steroid and immunosuppression & $1(1.90)$ & \\
\hline Topical and systemic steroid & $7(13)$ & \\
\hline
\end{tabular}

\section{Investigations to support the diagnosis of active or latent tuberculosis}

Data regarding the results of investigations arranged from the uveitis clinic are shown in Table 2. It is seen that a large proportion of patients (29) were referred for ATT for ocular TB mainly on the basis of a positive Mantoux and/or T-spot result, with normal chest X-ray imaging and no systemic symptoms.

\section{ATT regimens, duration and compliance}

Of the 54 patients in whom treatment was recommended, 7 (13\%) patients did not comply. The remaining 47 patients received a course of ATT. Duration of therapy 
Table 2 Investigations to support TB

\begin{tabular}{ll}
\hline & Number of patients \\
\hline No details available & 4 \\
Chest CT changes & 4 \\
$\quad$ With positive T-spot or Mantoux test & 3 \\
Positive Mantoux alone & 15 \\
Positive T-spot alone & 12 \\
Positive T-spot and Mantoux test & 2 \\
Typical X-ray changes present & 6 \\
$\quad$ With a positive T-spot and/or Mantoux test & 6 \\
Systemic symptoms present ${ }^{\text {a }}$ & 11 \\
$\quad$ With a positive T-spot and/or Mantoux test & 7 \\
With consistent CT or Chest X-ray findings & 3 \\
\hline
\end{tabular}

${ }^{a}$ One patient underwent a bone marrow biopsy which confirmed mycobacterium tuberculosis

ranged from 4 to 12 months with a mean of 6.8 months. Treatment regimens varied; however, all patients received rifampicin, with 9 receiving rifampicin, isoniazid and pyrazinamide (RHZ) and 27 received RHZ and ethambutol (E). See Table 3.

\section{Disease activity following completion of therapy}

Of the 47 patients who complied with ATT, follow-up data were available for 41 , with the remaining patients not attending follow-up or having missing data. Duration of clinic follow-up ranged from 20 to 2192 (median 557) days with five patients being lost to follow-up or who had moved elsewhere. Patients completing therapy were instructed to attend eye casualty in the event of flare-up, and it was assumed that their disease remained quiescent if no attendances were recorded. The mean clinic follow-up was 808 days.

A Kaplan-Meier survival curve for the 41 patients with post-ATT follow-up data is shown in Fig. 1a. The survival curve shows $90 \%$ of patients (39) were flare free 6 months

Table 3 Treatment regimens

\begin{tabular}{ll}
\hline$R$ alone & 2 \\
$R+E$ & 2 \\
$R+Z$ & 1 \\
$R H Z$ & 9 \\
$R E Z$ & 3 \\
$R H Z E$ & 27 \\
Anti-TB (unknown specific treatment) & 3 \\
Additional prednisone & 37 \\
Non-compliant & 7 \\
Mean duration of therapy & 6.8 months (range 4-12) \\
\hline
\end{tabular}

*Rifampicin $\mathrm{R}$, isoniazid $\mathrm{H}$, pyrazinamide $\mathrm{Z}$, ethambutol $\mathrm{E}$ following ATT treatment and 80\% (33) remained quiescent for 12 months.

A survival curve including patients for which both pre- and post-treatment data was available is shown in Fig. 1b. This highlights that $94 \%$ of patients were flare free at 6 months and $86 \%$ were flare free at 12 months.

The data set was then divided into two groups, those patients who were treated with ATT within 8 months of their first uveitis episode (group 1) and the remaining (group 2) who had had a diagnosis of uveitis for more than 8 months. This was to see whether patients with a longer duration of uveitis were less likely to respond favorably to ATT. Kaplan-Meier and log-rank tests (shown in Fig. 1c) were used for analysis. No significant difference in the time to flare was found ( $P=0.565$ log-rank) between the two groups.

The monthly flare rate pre-ATT treatment was calculated from the first uveitis episode to initiation of ATT. It was assumed the nine patients with a very short time to treatment $(<4$ months) would have had just one flare in the time period. Post-treatment flare rates were calculated from time of ATT completion to last day of data collection (December 2016). Flare rates after ATT were significantly lower than those before (Wilcoxon signed rank $P=0.000)$.

The data was further split into two groups; group 1 included patients treated within 8 months of their first uveitis flare. Group 2 included those who were treated more than 8 months after their first uveitis flare. Flare rates post-ATT treatment for both groups were significantly lower than pre-ATT (Wilcoxon signed rank $P=0.000$, $P=0.000$ respectively).

To assess whether the short time to treatment following first uveitis episode had an overall effect on the significant difference in pre- and post-ATT flare rates, the nine patients with less than 4 months follow-up were excluded and the data re-analysed. The post-ATT flare rates remaining significantly lower following the exclusion of these patients ( $P=0.000$ Wilcoxon signed rank). Of the nine patients excluded, all were flare free at 12 months.

Disease flare rates were also calculated using rates per person year (PPY). Prior to ATT treatment, the flare rate was 0.69 while after ATT, this reduced to 0.14 . The difference was statistically significant $(P=$ 0.000 mid- $P$ exact).

\section{Discussion}

The principal finding from this study is that ATT in a real-life clinical setting appears to significantly reduce the number of flare-ups and to enable long-term remission in patients with presumed ocular tuberculosis. This beneficial effect appears to persist even in patients who experienced a long delay between the onset of uveitis and the diagnosis of ocular TB. 

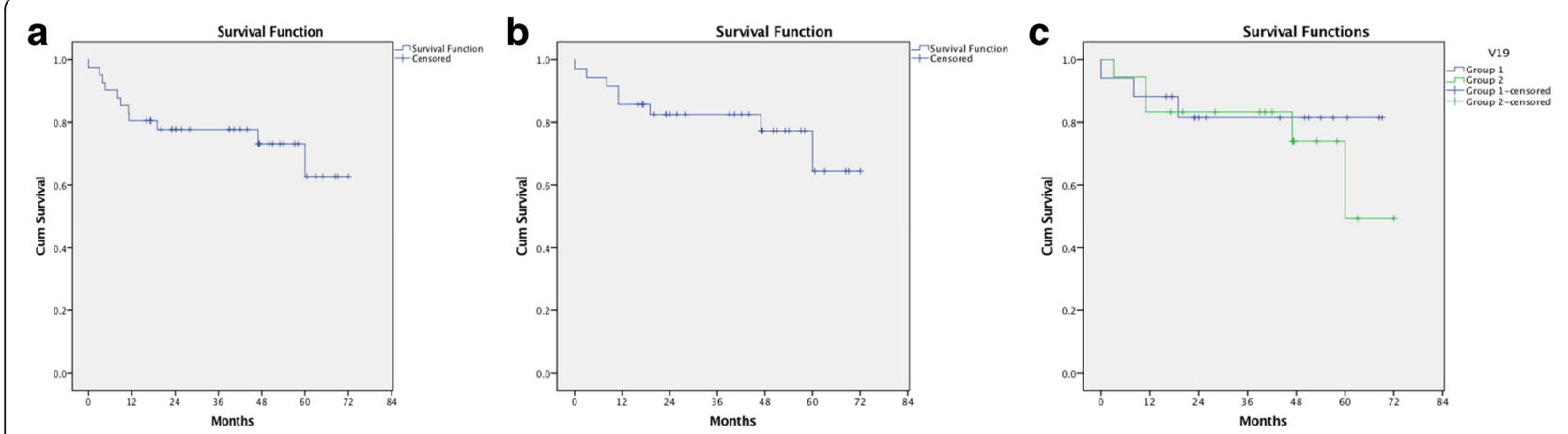

Fig. 1 Kaplan-Meier Survival curves showing: (a) time to flare-up for patients with post ATT followup data, (b) time to flare-up for patients with both pre and post ATT data and (c) time to flare-up for patients in group 1 and group 2 where group 1 were treated with ATT within 8 months of their first uveitis attack and group 2 were treated more than 8 months after their first uveitis attack

The patient cohort is diverse and strict diagnostic criteria are absent, requiring a high degree of clinical judgment and collaboration between specialists when treating such patients.

The study has limitations in view of the "real-life" clinical setting. Many were lost to follow-up or non-compliant with ATT. "Flare rate" was used as a measure of disease activity but may not accurately reflect disease in patients with ongoing activity, where uveitis is chronically uncontrolled, or being suppressed with corticosteroid. ATT was not standardized, and consequently, patients received a range of regimens. The majority however received at least 6 months of therapy. There is evidence to support treating patients for at least 9 months [29] although a wide range of treatment regimens and durations is reported in the literature.

Recent studies investigate therapeutic outcomes and the beneficial effects of ATT. One meta-analysis concluded that ATT in the management of patients with presumed ocular TB enabled remission in 84\% [30]. Another large analysis explored whether any factors were associated with a poorer outcome and found that prior immunosuppression and/or a high absolute QuantiFERON GOLD level were associated with poorer resolution of inflammation post-ATT or an ongoing need for systemic steroid [34].

This study highlights challenges and controversies in defining outcomes in such cohorts. We attempted to look at therapeutic outcomes in a number of ways, including comparing flare rates pre and post, reporting time to flare using Kaplan-Meier survival curves and also calculating "flare rates" before and after treatment. We also recognize that such measures may introduce bias and favour a benefit in patients with a short period of disease prior to the use of ATT. For this reason, we compared results between patients with a long duration of disease and a shorter duration of disease prior to ATT. Despite some patients experiencing significant delays in being started on ATT, we showed that such patient still displayed a clear benefit from treatment.

We did not report visual outcomes or complications. Whilst we show that there is a significant treatment benefit, even when duration of uveitis is long, patients with longer disease courses prior to ATT will be more likely to suffer visual loss and complications. Therefore, early treatment should be recommended [35].

\section{Conclusions}

In conclusion, this retrospective study shows that despite the unresolved controversies regarding diagnosis, the relevance of interferon gamma assays and what exactly ocular tuberculosis is, if patients have a consistent uveitis phenotype and other uveitic causes are excluded, then ATT is likely to have a beneficial effect.

\section{Acknowledgements}

We would like to acknowledge Peter Nightingale for his assistance with the statistical analysis, Dr. Nazim Nathani, Miss Sahar Al Hussainy, Dr. Guy Hagan and Miss Panagiota Stavrou for their involvement in the clinical care of these patients.

\section{Funding}

No funding resources were used for this research project.

\section{Authors contributions}

EMD conceived the original design, supervised data collection and statistical analysis and wrote the manuscript. SD participated in data collection and statistical analysis and contributed to the manuscript. CM participated in data collection and contributed to the manuscript. $\mathrm{XL}$ and $\mathrm{JH}$ participated in data collection and review of the manuscript. AD, EM, MD and PIM participated in clinical care of the patients, study design and review of the manuscript. All authors read and approved the final manuscript.

\section{Ethics approval and consent to participate}

This study used retrospective observational clinical data. Ethical approval was not required as this was deemed to be an evaluation of service. 


\section{Publisher's Note}

Springer Nature remains neutral with regard to jurisdictional claims in published maps and institutional affiliations.

\begin{abstract}
Author details
'Birmingham and Midland Eye Centre, City Hospital, Sandwell and West Birmingham Hospitals NHS Trust, Birmingham B18 7QH, UK. ${ }^{2}$ Sandwell General Hospital, Lyndon, West Bromwich, West Midlands B71 4HJ, UK. ${ }^{3}$ Department of Ophthalmology, Queen Elizabeth Hospital Birmingham, University Hospitals Birmingham NHS Foundation Trust, Birmingham, UK. ${ }^{4}$ Institute of Inflammation and Ageing, College of Medical and Dental Sciences, University of Birmingham, Edgbaston, Birmingham B15 2TT, UK. ${ }^{5}$ Heartlands Hospital, University Hospital Birmingham NHS Foundation Trust, Birmingham, UK. ${ }^{6}$ Birmingham Chest Clinic, University Hospitals Birmingham NHS Foundation Trust, Birmingham, UK.
\end{abstract}

\section{Received: 19 September 2017 Accepted: 20 November 2017} Published online: 04 December 2017

\section{References}

1. SUN WORKING GROUP (2005) Standardization of uveitis nomenclature for reporting clinical data. Results of the first international workshop. Am J Ophthalmol 140(3):509-516. doi:10.1016/j.ajo.2005.03.057

2. Deschenes J, Murray PI, Rao NA, Nussenblatt RB (2008) International Uveitis Study Group International Uveitis Study Groupd (IUSG): clinical classification of uveitis. Ocul Immunol Inflamm 16(1):1-2.

3. Durrani OM, Tehrani NN, Marr JE, Moradi P, Stavrou P, Murray PI (2004) Degree, duration, and causes of visual loss in uveitis. Br J Ophthalmol 88 : 1159-1162. doi:10.1136/bjo.2003.037226

4. de Smet MD, Taylor SRJ, Bodaghi B et al (2011) Understanding uveitis: the impact of research on visual outcomes. Prog Retin Eye Res 30(6):452-470. doi:10.1016/j.preteyeres.2011.06.005

5. Davidson M (1929) The modern approach to the problem of ocula tuberculosis: Werdenberg, E. Schweiz. Am J Ophthalmol 59(6):126

6. Al-Baker ZM, Bodaghi B, Khan SA (2016) Clinical patterns and causes of uveitis in a referral eye clinic in Qatar. Ocul Immunol Inflamm 0(0):1-10. doi:10.1080/09273948.2016.1206573

7. Sabhapandit S, Murthy SI, Singh VM et al (2016) Epidemiology and clinical features of uveitis from urban populations in South India. Ocul Immuno Inflamm 0(0):1-7. doi:10.1080/09273948.2016.1236971

8. Dogra M, Singh R, Agarwal A et al (2016) Epidemiology of uveitis in a tertiary-care referral institute in North India. Ocul Immunol Inflamm 0(0):1-8 doi:10.1080/09273948.2016.1255761

9. Nguyen M, Siak J, Chee S-P, Diem VQH (2016) The spectrum of uveitis in Southern Vietnam. Ocul Immunol Inflamm 0(0):1-7. doi:10.1080/09273948. 2016.1231826

10. Wong A, McKelvie J, Slight C, Sims J (2017) Land of the Long White Cloud: The Spectrum of Uveitis at a Tertiary Referral Center in New Zealand. Ocul Immunol Inflamm 25(sup1):S115-S121.

11. Jones NP (2015) The Manchester Uveitis Clinic: the first 3000 patients—epidemiology and casemix. Ocul Immunol Inflamm 23(2):118-126. doi:10.3109/09273948.2013.855799

12. Levy-Clarke G, Jabs DA, Read RW, Rosenbaum JT, Vitale A, Van Gelder RN (2014) Expert panel recommendations for the use of anti-tumor necrosis factor biologic agents in patients with ocular inflammatory disorders. Ophthalmology 121(3):785-796. doi:10.1016/j.ophtha.2013.09.048

13. Jabs DA, Rosenbaum JT, Foster CS et al (2000) Guidelines for the use of immunosuppressive drugs in patients with ocular inflammatory disorders: recommendations of an expert panel. Am J Ophthalmol 130(4):492-513 Available at: http://www.ncbi.nlm.nih.gov/pubmed/11024423

14. Tomkins-Netzer $O$, Talat $L$, Bar A et al (2014) Long-term clinical outcome and causes of vision loss in patients with uveitis. Ophthalmology 121(12): 2387-2392. doi:10.1016/j.ophtha.2014.07.007

15. Varma D, Anand S, Reddy AR et al., Tuberculosis: an under-diagnosed aetiological agent in uveitis with an effective treatment. Eye (Lond) 20(9): 1068-1073 doi:10.1038/sj.eye.6702):1068-73. doi:10.1038/sj.eye.6702093

16. Singh R, Toor P, Parchand S, Sharma K, Gupta V, Gupta A (2012) Quantitative polymerase chain reaction for mycobacterium tuberculosis in so-called Eales' disease. Ocul Immunol Inflamm 20(3):153-157. doi:10.3109/09273948. 2012.658134
17. Singh U, Mohapatra S, Wagh V, Porwal C, Kaushik A, Rajpal (2015) Association of mycobacterium tuberculosis in the causation of Eales' disease: an institutional experience. Indian J Med Microbiol 33(5):43. doi:10.4103/0255-0857.148829

18. Bansal R, Sharma K, Gupta A et al (2015) Detection of mycobacterium tuberculosis genome in vitreous fluid of eyes with multifocal serpiginoid choroiditis. Ophthalmology 122(4):840-850. doi:10.1016/j.ophtha.2014.11.021

19. Biswas J, Kazi M, Agarwal V, Alam MS, KI T (2016) Polymerase chain reaction for mycobacterium tuberculosis DNA detection from ocular fluids in patients with various types of choroiditis in a referral eye center in India. Indian J Ophthalmol 64(12):904. doi:10.4103/0301-4738.198857

20. Bhagya S, Lalitha P, Kumar AL, Rathinam S (2017) Polymerase chain reaction and its correlation with clinical features and treatment response in tubercular uveitis. Ocul Immunol Inflamm 0(0):1-8. doi:10.1080/09273948. 2017.1287925

21. Ang M, Wong W, Ngan CCL, Chee S-P (2012) Interferon-gamma release assay as a diagnostic test for tuberculosis-associated uveitis. Eye (Lond) 26(5):658-665. doi:10.1038/eye.2012.1

22. Cordero-Coma M, Calleja S, Torres HE et al (2010) The value of an immune response to mycobacterium tuberculosis in patients with chronic posterior uveitis revisited: utility of the new IGRAs. Eye (Lond) 24(1):36-43. doi:10.1038/eye.2009.51

23. Ang M, Chee S-P (2017) Controversies in ocular tuberculosis. Br J Ophthalmol 101(1):6-9. doi:10.1136/bjophthalmol-2016-309531

24. Bramante CT, Talbot E a, Rathinam SR, Stevens R, Zegans ME (2007) Diagnosis of ocular tuberculosis: a role for new testing modalities? Int Ophthalmol Clin 47(3):45-62. doi:10.1097/IO.0b013e318074de79

25. Ang M, Vasconcelos-Santos DV, Sharma K et al (2016) Diagnosis of ocular tuberculosis. Ocul Immunol Inflamm 3948(July):1-9. doi:10.1080/09273948. 2016.1178304

26. Ang M, Htoon HM, Chee SP (2009) Diagnosis of tuberculous uveitis: clinical application of an interferon-gamma. OPHTHA 116(7):1391-1396. doi:10.1016/j.ophtha.2009.02.005

27. Bansal R, Gupta A, Gupta V, Dogra MR, Bambery P, Arora SK (2008) Role of anti-tubercular therapy in uveitis with latent/manifest tuberculosis. Am J Ophthalmol 146(5). doi:10.1016/j.ajo.2008.06.011

28. Agrawal R, Gupta B, Gonzalez-lopez FJJ, et al. The role of anti-tubercular therapy in patients with presumed ocular tuberculosis. 2015;23(October 2014):40-46. doi:10.3109/09273948.2014.986584

29. Ang M, Hedayatfar A, Wong W, Chee S-P (2012) Duration of anti-tubercular therapy in uveitis associated with latent tuberculosis: a case-control study. $\mathrm{Br}$ J Ophthalmol 96(3):332-336. doi:10.1136/bjophthalmol-2011-300209

30. Kee AR, Gonzalez-lopez JJ, Al-hity A et al (2016) Anti-tubercular therapy for intraocular tuberculosis: a systematic review and meta- analysis. Surv Ophthalmol 61(5):628-653. doi:10.1016/j.survophthal.2016.03.001.This

31. Lou SM, Larkin KL, Winthrop K, Rosenbaum JT (2015) Lack of consensus in the diagnosis and treatment for ocular tuberculosis among uveitis specialists Ocul Immunol Inflamm 23(1):25-31. doi:10.3109/09273948.2014.926936

32. Conant MM, Vrasich CR, Wongskhaluang J V, et al. Role of the infectious disease consultant in management of patients with tuberculosis-associated ocular inflammation. 2015:1-5. doi:10.1093/ofid/ofv195

33. Keane J, Gershon S, Wise R, et al. Tuberculosis associated with Infliximab,. 2001;345(15):1098-1104.

34. Agrawal R, Gonzalez-Lopez J, Nobre-Cardoso J et al (2013) Predictive factors for treatment failure in patients with presumed ocular tuberculosis in an area of low endemic prevalence. $\mathrm{Br} J$ Ophthalmol 17(11):545149. doi:10.1136/bjophthalmol-2014-306474

35. Gunasekeran DV, Gupta B, Cardoso J, Pavesio CE, Agrawal R (2017) Visual morbidity and ocular complications in presumed intraocular tuberculosis: an analysis of 354 cases from a non-endemic population. Ocul Immunol Inflamm 0(0):1-5. doi:10.1080/09273948.2017.1296580 